\title{
Thanks to our reviewers
}

Published online: 2 May 2014

(C) Springer-Verlag Berlin Heidelberg 2014

On behalf of the entire editorial staff (co-editors, review editors, editorial board and Springer SMO publication and production team), I want to thank the following reviewers for their great help in maintaining and improving the quality of the journal in 2013.

George Rozvany

Editor-in-Chief
N. Aage
M. Abbasi
M. Abdalla
K. Abe
E. Acar
S. Adriaenssens
S. M. Afonso
J. Agte
A. Aguiar Gomes
J. Ahn
M. Akgun
N. Alagumurthy
N. Alexandrov
D. Allaire
A. Allen
J. Allison
J. Ambrosio
O. Amir
S. Amstutz
H. Andrae
C. Andreasen
E. Andreassen
R. Ansola
C. Antonio
M. Arakawa

A. Araujo

J.-M. Bourinet

U. Armani

P. Breitkopf

A. Asadpoure

M. Bruggi

T. Atanackovic

T. Bruns

V. Aute

R. Averill

H. Azegami

M. Bruyneel

D. Bae

H.-R. Bae

W. Baker

V. Balabanov

A. Baldini

J. Barradas Cardoso

M. H. Barros

R. Bulatovic

T. Burczynski

M. Burger

R. Butler

F.-J. Barthold

A. Basudhar

S. Bates

A. Beck

M. Berggren

D. Bettebghor

R. Bhatt

Z. Bingul

P. Block

M. Blosser

M. Bogomolny

P. Boisse

M. Campbell

A. Canelas

R. Canfield

Y. Caniou

Z. Cao

P. Carlsson

M. Carrasco

E. Castillo

P. Cha

C.-M. Chan

N. Chandiramani

A. Chateauneuf

A. Chaudhuri

L. Chen

S. Chen

S. Chen

X. Chen 
Y. Chen

Y. Chen

C. Chevalier

C. Chevallereau

M. Cho

J.-H. Choi

H.-L. Choi

S. Choi

S. Chowdhury

S. Choze

C.-H. Chuang

M. Clerc

P. Coelho

P. Coffin

M.C. Constantinou

K. Craig

E. Cramer

G. Craveur

K. Dalbey

M. Dambrine

M. Daskilweicz

T. Davis

A. de Faria

F. de Gournay

M. de Matos Neves

J. Deaton

K. Deb

Y. Deqing

T. Dersjö

J.-A. Desideri

M. Diez

Z. Dimitrovová

M. Domaszewski

J. Dong

R. dos Santos

J. Du

F. Duddeck

I. Elishakoff

R. Enblom

T. Erfani

A. Eriksson

P. Etman

A. Evgrafov

E. Fancello

H. Fang

S. Ferguson

R. Filomeno Coelho

J. Folgado

J. Fonseca
M. Fragiadakis

A. Friedlander

Y. Fu

K. Fuchi

F. Fuerle

J. Gablonsky

R. Ganguli

T. Gao

N. Gaul

A. Gaynor

G. Georgiou

B. German

H. Ghiasi

M. Gilbert

A. Giunta

M. Gobbi

T. Goel

C. Gogu

S. Gonella

Y. Gong

A. Goupee

G. Gray

L. Green

I. Grindeanu

Z. Gürdal

H. Gutierrez

S.-H. Ha

Y. D. Ha

A. Habbal

A. Hahn

J. Hamel

K. Hamza

J. Han

B. Hancock

A. Haque

A. Harte

B. Hassani

J. Herencia

S. Hernández

M. Hojjat

E. Holmberg

Y. Hongling

B. Horowitz

B. Houska

C. Hoyle

G. Hrinda

C. $\mathrm{Hu}$

J. $\mathrm{Hu}$

J. Hu

W. Hu

Z. $\mathrm{Hu}$
G. H. Huang

H. Huang

M. Hull

M. Hussein

M. Innocente

M. Jalalpour

D. Janjic

J. Jensen

G. Jivotovski

F. Jouve

A. Kale

B.-S. Kang

C.-H. Kang

Z. Kang

Y. Kanno

R. Kapania

C. Kassapoglou

A. Kaveh

A. Kawamoto

A. Keane

G. Kennedy

K. Khandelwal

H. Kim

I. Y. Kim

J.-H. Kim

Y. Y. Kim

T. Kipouros

S. Kitayama

A. Klarbring

P. Koch

M. Kocvara

R. Kolonay

R. Korycki

V. Kreinovich

J. Kruzelecki

A. Kulkarni

M. Kurdi

H. Kurtaran

L. Lamberti

M. Langelaar

O. Lavan

B. Lazarov

C. Le

C. Lee

D.-K. Lee

E. Lee

I. Lee

J. Lee

J. Lee

J. Lee 
S.-J. Lee

S. Lee

T. H. Lee

D. Lepadatu

$\mathrm{K}$. Lewis

T. Lewinski

G. Li

H. Li

M. Li

Q. Li

W. Li

C. W. Lim

P. T. Lin

Z. Lin

E. Lindgaard

L. Liu

S. Liu

Z. Liu

J. Lopez

X. Lu

Y. Lu

Z. $\mathrm{Lu}$

E. Lund

Z. Luo

H. Ma

Z.-D. Ma

C. Mader

S. Mahadevan

R. Malak

V. Mallardo

G. Marano

K. Marhadi

M. Marinaki

R. Marler

K. Marti

J. Martin

T. Martin

T. Matsumura

A. Mazurek

J. Meaders

Y. Mei

G. Melo

B. Mesmer

A. Micheletti

S. Min

S. Missoum

B. Mohammadi

M. Moore

A. Morris

Z. Mourelatos

D. Mueller
A. Mugan

V. Mukhopadhyay

A. Mullur

T. Nagayama

A. Nagy

Y. Narita

S. Nayak

L. $\mathrm{Ng}$

N.-V. Nguyen

T. Nguyen

T. Nikbay

E. Nikolaidis

E. Nikolaidis

L. Nilsson

S. Nishiwaki

B. Niu

T. Nomura

W. I. Notz

A. Novotny

J. Ochsendorf

M. Ohsaki

R. Ouedraogo

M. Paas

S. Padula

M. Pais

J. Pajot

R. Pan

S. Pantelakis

O. Pantz

S. Parashar

C. Park

G. Paulino

N. Paya-Zaforteza

C. Pedersen

N. Pedersen

R. Perez

V. Picheny

A. Pichugin

G. Pingen

M. Price

X. Qian

N. Queipo

M. Rais-Rohani

P. Ramu

A. R. M. Rao

P. Rao

V. Rao
T. Ray

M. Reed

J. Reinbolt

B. Reza

B. O. Rhee

M. Riley

N. Roberty

J. Robinson

J. R. Roche

D. Romero

V. J. Romero

O. Roustant

M. P. Saka

B. Sankar

M. Schevenels

U. Schramm

C. Seeley

M. Shimoda

S. Siddiqui

E. Silva

T. Simpson

A. Sobester

T. Sokol

J. Sokolowski

C. Song

C. Y. Song

H. Song

J.-H. Song

M. Sönmez

C. Soto

A. Srikanth

K. Srinivas

N. Stander

G. Steven

M. Stingl

M. Stolpe

R. Su

G. Subhash

A. Suleman

G. Sun

J.-Q. Sun

Y. Sung

K. Suresh

S. Szyniszewski

Y. Tada

K. Tai

I. Takewaki

C. Talischi

A. Tekalur

J. A. Tenreiro Machado 
E. Thompson

A. Thrall

G. Tibert

A.-M. Toader

D. Toal

A. Todoroki

M. Tootkaboni

A. Tovar

R. Troian

K.-Y. Tseng

I. Tsukanov

L. Tudose

S. Turteltaub

M. Utz

U. K. Vaidy

N. van Dijk

L. Van Miegroet

P. Vannucci

K. Venkatesh Raja

G. Venter

N. Vermaak

F. Vernerey

F. Viana

T. Vietor

D. Villanueva

A. Vincenti

R. Vogel
T. Wagner

B. Wang

B. P. Wang

G. G. Wang

L. Wang

L. Wang

N. Wang

P. Wang

W. Wang

X. Wang

J. Wannenburg

G. Waycaster

P. Weaver

P. Wei

F. Wein

A. Wood

H. X

Z. Xi

Q. Xia

N.-G. Xie

Y.-M. Xie

Y. Xiong

H. Xu

S. Xu

T. Yamada

S. Yamasaki

J. Yan
D. Yang

J. Yang

Y. Yang

J. Ye

V. Yepes

T. Yildiz

J. Yoo

G. H. Yoon

S.-K. Youn

S. Zein

D. Zeng

M. Zhai

J. Zhan

J. Zhang

V. Zhang

W. Zhang

X.-L. Zhang

Y. Zhang

G. Zhao

L. Zhao

K. Zhou

Q. Zhou

S. Zhou

J.-H. Zhu

P. Zhu

X. Zhu

D. Zingg

A. Zmitrowicz 